\title{
Analytic solutions of the diffusion-deposition equation for fluids heavier than atmospheric air
}

\author{
F. P. Barrera ${ }^{1}$, T. Brugarino ${ }^{2}$, V. Piazza $^{3} \&$ L. Pignato $^{3}$ \\ ${ }^{1}$ Dip. di Ingegneria dei Trasporti, \\ Università di Palermo Facoltà d'Ingegneria, 90128 Palermo, Italy \\ ${ }^{2}$ Dip. di Metodi e Modelli Matematici, \\ Università di Palermo Facoltà d'Ingegneria, 90128 Palermo, Italy \\ ${ }^{3}$ Dip. di Ricerche Energetiche ed Ambientali \\ Università di Palermo Facoltà d'Ingegneria, 90128 Palermo, Italy
}

\begin{abstract}
A steady-state bi-dimensional turbulent diffusion equation was studied to find the concentration distribution of a pollutant near the ground. We have considered the air pollutant emitted from an elevated point source in the lower atmosphere in adiabatic conditions. The wind velocity and diffusion coefficient are given by power laws. We have found analytical solutions using or the Lie Group Analysis or the Method of Separation of Variables. The classical diffusion equation has been modified introducing the falling term with non-zero deposition velocity.

Analytical solutions are essential to test numerical models for the great difficulty in validating with experiments.

Keywords: atmospheric pollution, diffusion equation, exact solutions.
\end{abstract}

\section{Introduction}

The classical form of the mean steady diffusion equation is valid for elementary particles of the fluid or when the foreign particles are of the same density as the fluid. If the density and dimensions are high enough to have terminal velocities $v_{s}$ not negligible, the distribution of the particles will be affected in various ways [1-4].

A simple approximation is to consider that the particle sinks at a rate $v_{s}$ and the ground acts as a permeable surface and retains all material passing through it.

Using a very simple model it is possible to examine various cases. It is possible to have exact solutions of the mean steady diffusion when the turbulent diffusivity $k_{z}$ and the terminal velocity $v_{d}$ depend somehow on the height [5-7]. 


\section{Mathematical model}

The sedimentation of the material may be allowed by introducing a convection term in the mean steady equation that becomes $[8,9]$ :

$$
u(z) \frac{\partial c}{\partial x}=\frac{\partial}{\partial z}\left(k_{z}(z) \frac{\partial c}{\partial z}+v_{s}(z) c\right)
$$

where $v_{s}(z)$ is the deposition velocity.

We assume that mean wind velocity $u(z)$, the eddy diffusivity in $z$-direction $k_{z}(z)$ and the deposition velocity $v_{s}(z)$ are:

$$
\begin{aligned}
u(z) & =u_{0} z^{\alpha} \\
k_{z}(z) & =k_{0} z^{n} \\
v_{s}(z) & =v_{0} z^{q}
\end{aligned}
$$

\section{Group analysis of the equation}

Group analysis of the (1) is performed through the one-parameter Lie group of transformations:

$$
\left\{\begin{array}{l}
x^{*}=x+\epsilon X(x, z, c)+O\left(\epsilon^{2}\right) \\
z^{*}=z+\epsilon Z(x, z, c)+O\left(\epsilon^{2}\right) \\
c^{*}=c+\epsilon C(x, z, c)+O\left(\epsilon^{2}\right)
\end{array}\right.
$$

where $X, Z, C$ are the infinitesimal generators of the transformations [10-12].

Equation (1) is invariant respect to the group (5) of transformations if $c^{*}$ is the solution of eq. (1) in the star variables. In this case, the number of independent variables can be decreased.

A considerable difficulty lies in the amount of the auxiliary calculations involved. We performed the calculations of the generators of the transformations group on a P.C. using the MATHEMATICA package.

Since eq. (1) is linear, the infinitesimal generators of the group of invariance are of the form:

$$
\left\{\begin{array}{l}
X=X(x) \\
Z=Z(x, z) \\
C=A(x, z) c+B(x, z)
\end{array}\right.
$$

The function $B(x, z)$ must satisfy eq. (1) and, without compromising with the generality, can be assumed equal to zero.

If we normalize the parameters $u_{0} / k_{0} \rightarrow v_{0}$ and the variable $k_{0} x / v_{0} \rightarrow x$, we have that $X, Z$ and $A$ must satisfy the following equations:

$$
n z^{-1} Z-z^{-1} \alpha Z+X^{\prime}-2 Z_{z}=0
$$




$$
\begin{gathered}
q v_{0} z^{-2-n+q} Z+n q v_{0} z^{-2-n+q} Z-q^{2} v_{0} z^{-2-n+q} Z-n z^{-1} A_{z} \\
-v_{0} z^{-n+q} A_{z}-2 q v_{0} z^{-1-n+q} Z_{z}-A_{z z}+z^{-n+\alpha} A_{x}=0 \\
n z^{-2} Z+n v_{0} z^{-1-n+q} Z-q v_{0} z^{-1-n+q} Z-2 A_{z}-n z^{-1} Z_{z} \\
-v_{0} z^{-n+q} Z_{z}+Z_{z z}-z^{-n+\alpha} Z_{x}=0
\end{gathered}
$$

We show now some results.

\section{Similarity solutions}

Let us look at some similarity solutions.

\section{1 $\alpha, n$ and $q$ arbitrary $(n-\alpha \neq 2)$}

In this case it possible to obtain from the eq.s (7-9) the generators of group of similarity:

$$
\left\{\begin{array}{l}
X=a_{0} \\
Z=0 \\
C=c_{1} c
\end{array}\right.
$$

where $a_{0}$, and $c_{1}$ are arbitrary constants.

The characteristic equations are:

$$
\frac{\mathrm{d} x}{x_{1}}=\frac{\mathrm{d} z}{0}=\frac{\mathrm{d} c}{c_{1} c}
$$

The invariants are $z$ and $c \mathrm{e}^{-\frac{c_{1}}{x_{1}} x}$. If we assume $\frac{c_{1}}{x_{1}}=-\lambda^{2}$, the similarity solution, corresponding to the separation of variables, is

$$
c=\mathrm{e}^{-\lambda^{2} x} Z(z)
$$

where $Z(z)$ is solution of the following ordinary differential equation:

$$
\left(q v_{0} z^{1-n+q}+z^{2-n+\alpha} \lambda^{2}\right) Z(z)+z\left(n+v_{0} z^{1-n+q}\right) Z^{\prime}(z)+z^{2} Z^{\prime \prime}(z)=0
$$

If $n=2 q-\alpha$, the solution is [13]

$$
\begin{aligned}
Z(z)= & \mathrm{e}^{\frac{z^{1-q+\alpha}\left(v_{0}+\sqrt{v_{0}^{2}-4 \lambda^{2}}\right)}{-2+2 q-2 \alpha}} \\
\times & {\left[h_{1} \Psi\left(\frac{2-3 q+2 \alpha-\frac{q v_{0}}{\sqrt{v_{0}^{2}-4 \lambda^{2}}}}{2(1-q+\alpha)}, \frac{2-3 q+2 \alpha}{1-q+\alpha} ; \frac{z^{1-q+\alpha} \sqrt{v_{0}^{2}-4 \lambda^{2}}}{1-q+\alpha}\right)\right.} \\
& \left.+h_{2} L\left(\frac{2-3 q+2 \alpha-\frac{q v_{0}}{\sqrt{v_{0}^{2}-4 \lambda^{2}}}}{-2(1-q+\alpha)}, \frac{1-2 q+\alpha}{1-q+\alpha} ; \frac{z^{1-q+\alpha} \sqrt{v_{0}^{2}-4 \lambda^{2}}}{1-q+\alpha}\right)\right]
\end{aligned}
$$




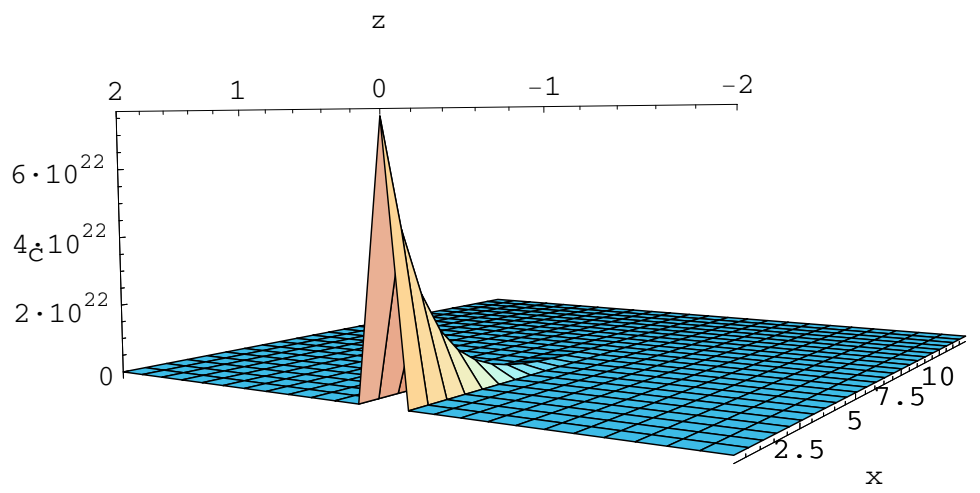

Figure 1: The $c(x, z), v_{0}=20, q=0.5, \lambda=1, \alpha=1.5, h_{1}=1, h_{2}=0$.

where $h_{1}$ and $h_{2}$ are arbitrary constants, $\Psi(-,-; \cdot)$ is the confluent hypergeometric function and $L(-,-; \cdot)$ is the generalized Laguerre polynomial. The concentration is

$$
\begin{aligned}
c= & \mathrm{e}^{-\lambda^{2} x} \mathrm{e}^{-\frac{\left(v_{0}+\sqrt{v_{0}^{2}-4 \lambda^{2}}\right)}{1-q+\alpha} \frac{z^{1-q+\alpha}}{2}} \\
& \times\left[h_{1} \Psi\left(\frac{2-3 q+2 \alpha-\frac{q v_{0}}{\sqrt{v_{0}^{2}-4 \lambda^{2}}}}{2(1-q+\alpha)}, \frac{2-3 q+2 \alpha}{1-q+\alpha} ; \frac{z^{1-q+\alpha} \sqrt{v_{0}^{2}-4 \lambda^{2}}}{1-q+\alpha}\right)\right. \\
& \left.+h_{2} L\left(\frac{2-3 q+2 \alpha-\frac{q v_{0}}{\sqrt{v_{0}^{2}-4 \lambda^{2}}}}{-2(1-q+\alpha)}, \frac{1-2 q+\alpha}{1-q+\alpha} ; \frac{z^{1-q+\alpha} \sqrt{v_{0}^{2}-4 \lambda^{2}}}{1-q+\alpha}\right)\right]
\end{aligned}
$$

\section{2 $n=1, q=0$ and $\alpha$ arbitrary}

We observe that in this case $k_{0}=k u_{*}$ where $k$ is the Von Karman constant and $u_{*}$ is the friction velocity.

The generators of group of similarity are:

$$
\left\{\begin{array}{l}
X=a_{0}+a_{1} x+\frac{a_{2}}{2} x^{2} \\
Z=\frac{a_{1}+a_{2} x}{1+\alpha} z+c_{0} z \\
C=\left(b_{0}+b_{1} z^{1+\alpha}+b_{1} x(1+\alpha)\left(1+v_{0}+\alpha\right)\right) c
\end{array}\right.
$$

where $a_{0}, a_{1}, a_{2}, b_{0}, b_{1}$ and $c_{0}$ are constants satisfying the conditions

$$
c_{0}\left(1+2 v_{0}+\alpha\right)=0, \quad a_{2}+2 b_{1}(1+\alpha)^{2}=0
$$

Now we consider the following subcases. 
4.2.1 $c_{0}=0, a_{2}=-2 b_{1}(1+\alpha)^{2}$ and $\alpha \neq-1$

The characteristic equations are:

$$
\frac{\mathrm{d} x}{x}=(1+\alpha) \frac{\mathrm{d} z}{z}=\frac{\mathrm{d} c}{c}
$$

The invariants are $\frac{c}{x}$ and $\xi=z x^{-\frac{1}{1+\alpha}}$; the concentration is

$$
c=x f(\xi)
$$

where $f(\xi)$ is solution of the following ordinary differential equation:

$$
\xi^{\alpha} f(\xi)-\left(1+v_{0}+\frac{\xi^{1+\alpha}}{1+\alpha}\right) f^{\prime}(\xi)-\xi f^{\prime \prime}(\xi)=0
$$

In this case we have:

$$
\begin{aligned}
f(\xi)=\mathrm{e}^{-\frac{z^{1+\alpha}}{x(1+\alpha)^{2}}}[ & h_{1} \Psi\left(\frac{2+v_{0}+2 \alpha}{1+\alpha}, 1+\frac{v_{0}}{1+\alpha} ; \frac{z^{1+\alpha}}{x(1+\alpha)^{2}}\right) \\
+ & \left.h_{2} L\left(-\frac{2+v_{0}+2 \alpha}{1+\alpha}, \frac{v_{0}}{1+\alpha} ; \frac{z^{1+\alpha}}{x(1+\alpha)^{2}}\right)\right]
\end{aligned}
$$

where $h_{1}$ and $h_{2}$ are arbitrary constants. The concentration is

$$
\begin{aligned}
c=x \mathrm{e}^{-\frac{z^{1+\alpha}}{x(1+\alpha)^{2}}} & {\left[h_{1} \Psi\left(\frac{2+v_{0}+2 \alpha}{1+\alpha}, 1+\frac{v_{0}}{1+\alpha} ; \frac{z^{1+\alpha}}{x(1+\alpha)^{2}}\right)\right.} \\
+ & \left.h_{2} L\left(-\frac{2+v_{0}+2 \alpha}{1+\alpha}, \frac{v_{0}}{1+\alpha} ; \frac{z^{1+\alpha}}{x(1+\alpha)^{2}}\right)\right]
\end{aligned}
$$

\subsection{2 $\left(1+2 v_{0}+\alpha\right)=0, a_{0}=-1, b_{1}=0$ and $\alpha \neq-1$}

The characteristic equations are:

$$
\frac{\mathrm{d} x}{-1}=\frac{\mathrm{d} z}{z^{\frac{1-\alpha}{2}}}=\frac{\mathrm{d} c}{c}
$$

The invariants are $c \mathrm{e}^{x}$ and $\xi=x+\frac{2}{1+\alpha} z^{\frac{\alpha+1}{2}}$; the concentration is

$$
c=\mathrm{e}^{-x} f(\xi)
$$

where $f(\xi)$ is solution of the following ordinary differential equation:

$$
f(\xi)-f^{\prime}(\xi)+f^{\prime \prime}(\xi)=0
$$

In this case the concentration is:

$$
f(\xi)=\mathrm{e}^{-\frac{x}{2}+\frac{\frac{1+\alpha}{2}}{1+\alpha}}\left[h_{1} \cos \sqrt{3}\left(\frac{x}{2}+\frac{z^{\frac{1+\alpha}{2}}}{1+\alpha}\right)+h_{2} \sin \sqrt{3}\left(\frac{x}{2}+\frac{z^{\frac{1+\alpha}{2}}}{1+\alpha}\right)\right]
$$




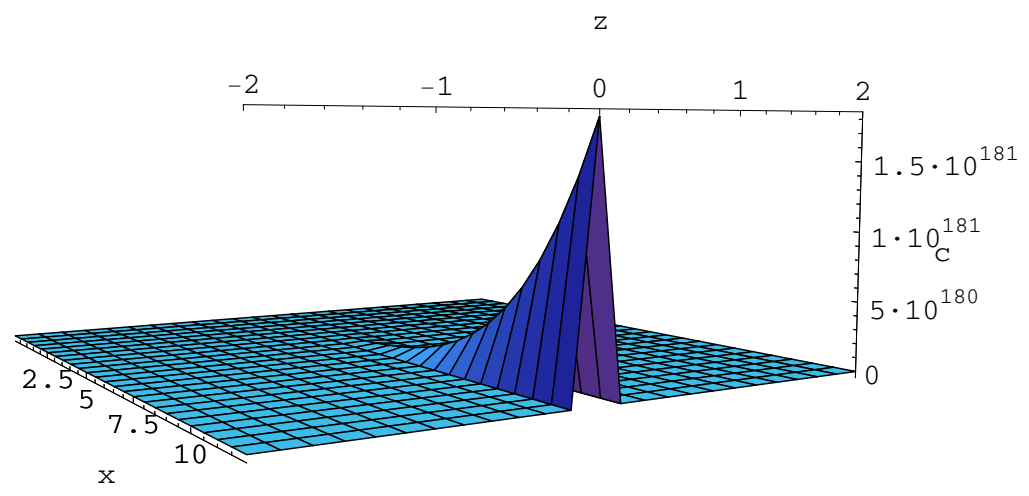

Figure 2: The $c(x, z), v_{0}=11, \alpha=1, h_{1}=1, h_{2}=0$.

\section{$4.3 n=2, q=1$ and $\alpha=0$}

The generators of group of similarity are:

$$
\left\{\begin{aligned}
X= & a_{0}+a_{1} x+\frac{a_{2}}{2} x^{2} \\
Z= & b_{0} z+\frac{1}{2}\left(a_{1}+a_{2} x\right) z \log z \\
C= & \left(c_{2}-\frac{1}{8} x\left(2 a_{1}\left(v_{0}-1\right)^{2}+a_{2}\left(2+\left(v_{0}-1\right)^{2} x\right)\right)\right. \\
& \left.-\frac{1}{8} \log z\left(2\left(v_{0}+1\right)\left(a_{1}+a_{2} x\right)+a_{2} \log z\right)\right) c
\end{aligned}\right.
$$

where $a_{0}, a_{1}, a_{2}, b_{0}$, and $c_{2}$ are arbitrary constants.

If we put: $a_{0}=0, a_{1}=1, a_{2}=0, b_{0}=0, c_{2}=0$, we have

$$
\left\{\begin{array}{l}
X=x \\
Z=\frac{1}{2} z \log z \\
C=-\frac{1}{4}\left(\left(v_{0}-1\right)^{2} x+\left(1+v_{0}\right) \log z\right) c
\end{array}\right.
$$

The invariants are $c \mathrm{e}^{\frac{1}{4}\left(v_{0}-1\right)^{2} x} z^{\frac{1}{2}\left(1+v_{0}\right)}$ and $\xi=\frac{\log z}{\sqrt{x}}$; the concentration is

$$
c=\mathrm{e}^{-\frac{1}{4}\left(v_{0}-1\right)^{2} x} z^{-\frac{1}{2}\left(1+v_{0}\right)} f(\xi)
$$

where $f(\xi)$ is solution of the following ordinary differential equation:

$$
f^{\prime \prime}(\xi)+\frac{\xi}{2} f^{\prime}(\xi)=0
$$




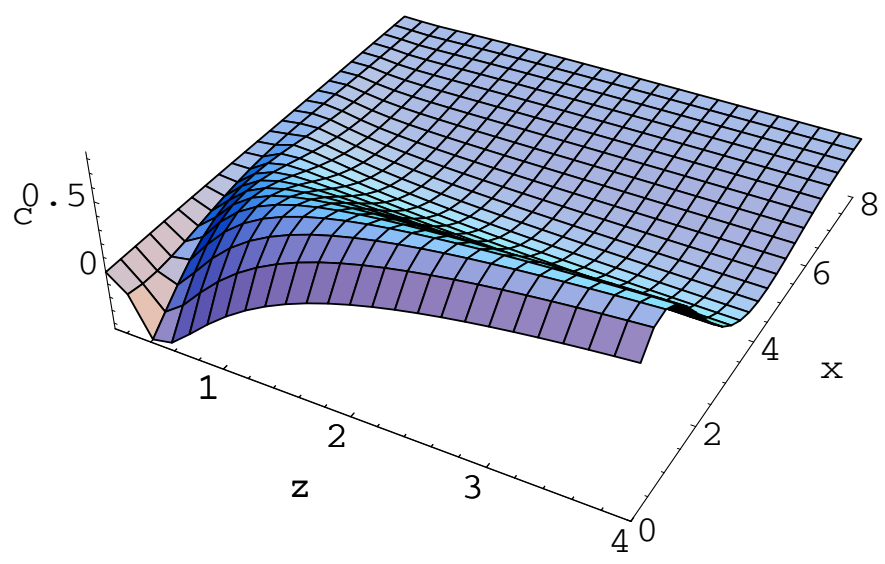

Figure 3: The $c(x, z), \alpha=-3, h_{1}=1, h_{2}=1$.

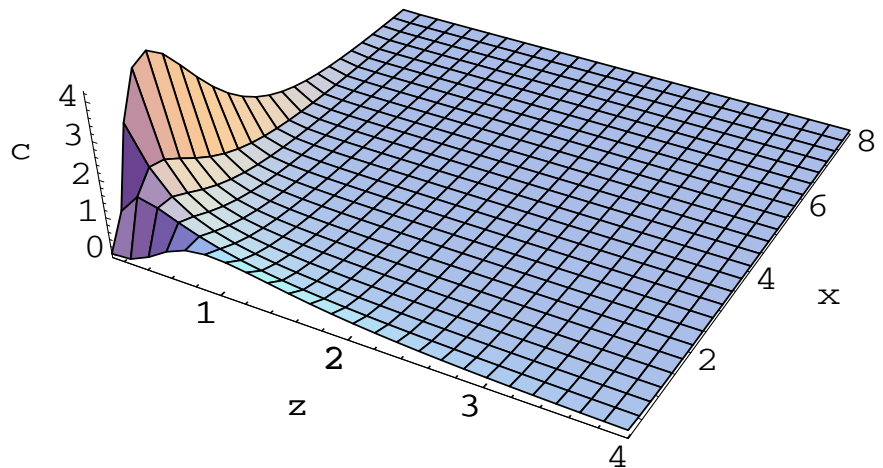

Figure 4: The $c(x, z), v_{0}=3, h_{1}=1, h_{2}=1$.

the solution is

$$
f(\xi)=h_{1}+h_{2} \operatorname{erf}\left(\frac{\xi}{2}\right)
$$

The concentration is

$$
c=\mathrm{e}^{-\frac{1}{4}\left(v_{0}-1\right)^{2} x} z^{-\frac{1}{2}\left(1+v_{0}\right)}\left(h_{1}+h_{2} \operatorname{erf}\left(\frac{1}{2} \frac{\log z}{\sqrt{x}}\right)\right)
$$




\section{Conclusion}

We obtain analytical solutions, using Lie group analysis, for steady-state bi-dimensional turbulent diffusion equation with variable coefficients. The laws of wind speed, turbulent diffusion coefficients and terminal velocities are specified by power laws. The obtained solutions are more realistic respect to gaussian model in air pollution modeling. In future we intend, using our solutions, to solve the diffusion equation (1) for many boundary conditions.

\section{References}

[1] Arya S. P., Air Pollution Meteorology and Dispersion Oxford University Press (1999).

[2] Pasquill F. and Smith F. B., Atmospheric Diffusion Ellis Horwood (1983).

[3] Seinfeld J. H., Atmospheric Chemistry and Physics of Air Pollution Wiley (1986).

[4] Sutton O. G., Micrometeorology M.Graw Hill (1953).

[5] Godson W. L., The diffusion of particulate matter from an elevated source; Archiv für Meteor. Geophys. Bioklim., Ser. A., 10, 305-327, (1958).

[6] Huang C. H., On solutions of the diffusion-deposition equation for point sources in turbulent shear flow; Journal of Applied Meteorology, 250-254, (1999).

[7] Rounds W., Solution of two dimensional diffusion equation; Trans. Amer. Geophys. Union, 36, 395-405, (1955).

[8] Yamamoto G., Shimamuki A., Nishinomiya S., Diffusion of falling particles in diabatic atmospheres; Journal of the Meteorological Society of Japan, 48, 417-424, (1970).

[9] Yeh G. T., Huang C. H., Three-dimensional air pollutant modeling in the lower atmosphere; Boundary Layer Meteorology, 9, 381-390, (1975).

[10] Barrera P., Brugarino T., Group analysis and some exact solution for the thermal boundary layer; Advances in Fluid Mechanics VI, WIT Press, 52, 327 337, (2006).

[11] Barrera P., Brugarino T., Some exact solutions of two coupled non linear diffusion-convection equations; Air Pollution XIII, Modelling, Monitoring and Management of Air Pollution, Cordova, (2005).

[12] Barrera P., Brugarino T., Pignato L., Solutions for a diffusion process in nonhomogeneous media; Il Nuovo Cimento B., Vol. 116 B, 8, 951-958, (2001).

[13] Polyanin A. D., Zaitsev V. F. Handbook of Exact Solutions for Ordinary Differential Equations CRC Press (1995). 